\title{
CAUSAS, MANIFESTACIONES Y EFECTOS DE LA VULNERACIÓN DEL DERECHO A LA SALUD EN LAS SENTENCIAS DE TUTELA DE LOS JUECES DE NEIVA
}

Doctor en Derecho Constitucional (U. de ParisFrancia) Docente investigador de la Universidad Surcolombiana. Director del Grupo Nuevas Visiones del Derecho, categoría B Colciencias.

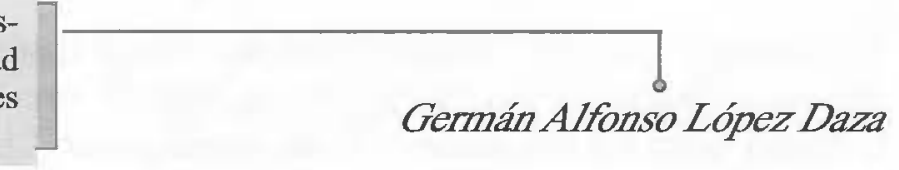

RESUMEN

A partir de la definición de obligaciones, principios y subsistemas relativos a la organización de la salud como prestación a cargo del Estado, en la Constitución Política de 1991 se consagró el derecho a la salud y a la seguridad social con múltiples elementos y formas de ejecución, que tienen por objetivo garantizar los fines del Estado Social de Derecho.

Actualmente, el desarrollo legal del sistema de seguridad social se encuentra contenido en forma detallada en la Ley 100 de 1993 modificada por la Ley 1122 del 9 de enero de 2007 y sus numerosos decretos y demás actos administrativos generales, que buscan cumplir los principios de eficiencia, universalidad`y solidaridad consagrados en el artículo 48 de la Constitución Política.

Durante estos 13 años de aplicación de la Ley 100 de 1993 han surgido diversas interpretaciones y por ende conflictos, lo que ha llevado a constitucionalizar la discusión y a que los jueces de instancia sean en últimas quienes decidan los alcances de la prestación del servicio de la salud.

En Colombia ya se han hecho investigaciones sobre esta problemática, cuyos análisis abarcan todo el país. Por ello, con el actual informe, se concreta la mirada sobre la ciudad de Neiva desde la perspectiva de la investigación - jurídica aplicada y a partir de las decisiones judiciales proferidas entre los años 2003 y 2004.

\section{PALABRAS CLAVE:}

Acción de tutela - Derecho a la salud - Constitucionalización del derecho - Interpretación judicial - Conexidad

\section{ABSTRACT}

From the definition of obligations, principles and subsystems relative to the organization of the health like benefit in charge of the State, in the Political Constitution of 1991 one devoted the right

* Informe parcial de la investigación jurídica desarrollada por el Grupo de Investigación Nuevas Visiones del Derecho de la Facultad de Derecho de la Universidad Surcolombiana, reconocido en Categoría B por Colciencias. 
to the health and the social security with multiple elements and forms of execution, that they must by objective guarantee the aims of the Social State of Right.

At the moment, the legal development of the social security system is content in form detailed in Law 100 of 1993 modified by Law 1122 of 9 of January of 2007 and its numerous decrees and other gęneral administrative acts that look for to fulfill the efficiency principles, consecrated universality and solidarity in article 48 of the Political Constitution.

During these 13 years of application of Law 100 of 1993 diverse interpretations and therefore conflicts have arisen, what he has taken to "constitucionalizar" the discussion and to that the instance judges are in last who decide the reaches of the benefit of the service of the health.

In Colombia already investigations have been made on this problematic one, whose analyses include all the country. For that reason, with the present report, the glance takes shape on the city of Neiva from the perspective of the investigation - legal applied and from the judicial decisions between years 2003 and 2004.

\section{KEYWORDS}

Action of trusteeship - Right to the health - "Constitucionalización" of the right - Judicial interpretation - Conexidad

\section{PROBLEMA DE INVESTIGACION}

Cuáles son los principales factores que permiten identificar las manifestaciones de la vulneración del derecho a la salud en las sentencias de tutela de los jueces de Neiva y cuáles son sus efectos en las instituciones que participan en esta problemática?

\section{OBJETIVO GENERAL}

Identificar y analizar las principales causas de vulneración del derecho fundamental a la salud, según los fallos de tutela proferidos por los jueces de Neiva, así como determinar los agentes que más inciden o generan dicha vulneración y las soluciones jurídicas que los jueces de tutela en Neiva han dado para esta problemática.

\section{DISEÑO METODOLÓGÍCO UNIVERSO DE ESTUDIO, SELECCIÓN, TAMAÑO DE MUESTRA Y DISEÑO DEL MUESTREO UNIDAD DE ANÁLISIS, HIPÓTESIS Y VARIABLES}

Teniendo en cuenta la problemática abordada, se considera que el enfoque epistemológico más conveniente a desarrollar en la presente investigaciónes el de la investigación descriptiva, puesto que comprende la descripción, el registro, el análisis y la interpretación de la situación actual de vulneración al derecho a la salud, la composición y los procesos de tal fenómeno.

Este enfoque se realizó sobre conclusiones dominantes en la actual caracterización, y sobre la forma en que los afectados y los prestatarios se comportan en la actualidad. Se parte de realidades fácticas determinadas por las decisiones judiciales locales. 
El estudio descriptivo incluye la metodología de análisis de casos, causales, de conjunto y de correlación.

\section{POBLACION}

1528 expedientes judiciales de acciones de tutela por vulneración del derecho a la salud, ubicados en los 48 despachos judiciales existentes en Neiva.

\section{RESULTADOS}

En adelante, metodológicamente se presentarán los resultados estadísticos de la investigación, ilustrados mediante las correspondientes tablas o gráficos, y posteriormente se hará una interpretación analítica de estos hallazgos, recordando que la investigación se limita temporalmente a los años 2003 y 2004 y espacialmente, a la ciudad de Neiva, en cuanto al derecho a la salud.

\subsection{Entidades Contra las Cũales se Formulala Acción de Tutela}

Se consideró infiportante establecer en primer lugar cuál es la entidad que según los registros estadísticos presenta mayores porcentajes en las acciones de tutela que se presentan en su contra, para de allí derivar cada uno de los elementos que configuran esta problemática regional, incluyendo los aspectos del prestatario, el usuario y el juzgador.

\section{Tabla 1. Entidad contra la cual se formula la acción de tutela}

\begin{tabular}{|l|c|c|}
\hline \multicolumn{1}{|c|}{ Entidad } & Frecuencia & Porcentaje \\
\hline 1. EPS SALUDCOOP & 473 & 31,0 \\
\hline 2. ISS EPS & 349 & 22,8 \\
\hline 3. CAFESALUD EPS & 161 & 10,5 \\
\hline 4. SECRESALUD DPTAL & 112 & 7,3 \\
\hline 5. HUMANA VIVIR SA EPS & 107 & 7,0 \\
\hline
\end{tabular}

\begin{tabular}{|l|c|c|}
\hline 6. CAJANAL & 091 & 6,0 \\
\hline 7. SOLSALUD SA EPS & 067 & 4,4 \\
\hline 8.CAJA SALUD & 038 & 2,6 \\
\hline 9. COOMEVA EPS & 022 & 1,4 \\
\hline 10. CAPRECOM & 017 & 1,1 \\
\hline 11. Otros & 91 & 6,2 \\
\hline 1otä & 1528 & 100,0 \\
\hline
\end{tabular}

* Con frecuencia entre $14(0,9 \%)$ y $1(0,1 \%)$ casos, se ubican en las siguientes entidades: Secretaría Municipal de Salud, Asmet Salud, Colsanitas, Emcosalud, Inpec, Salud Total, Sanitas Eps, Colseguros, Ecoopsos, Sanidad Policia, Colmena Salud, Arp Seguros La Equidad, Caja Agraria, Comsusalud, Cruz Blanca, Incora, Medicos Asociados S., Previsora Vidasalud, Prosalud Tolima, Salud Colmena, Sanidad Ejercito, Seguros La Equidad.

El 64,3\% de las acciones de tutela se dirigen, en cifras objetivas, contra tres entĩ dades, en su orden: 1). Saludcoop, 2.) ISS y 3.) Cafesalud. Sin embargo, a pesar de que SALUDCOOP EPS, empresa privada del sector solidario, aparece como la entidad con mayor número de tutelas formuladas en su contra, es necesario establecer la proporción respecto del número de afiliados de cada una de las entidades, según se detalla a continuación:

Tabla 1A. Afiliados a EPS y ARS 2003 - 2004

\begin{tabular}{|l|r|r|r|r|r|r|}
\hline \multicolumn{1}{|c|}{ Entidad } & $\begin{array}{c}2003 \\
\text { afiliados }\end{array}$ & $\begin{array}{c}2003 \\
\text { porcentaje }\end{array}$ & $\begin{array}{c}2004 \\
\text { afiliados }\end{array}$ & $\begin{array}{c}2004 \\
\text { porcentajee }\end{array}$ & $\begin{array}{l}\text { Promedio } \\
\text { afiliados }\end{array}$ & \% \\
\hline EPS SALUDCOOP & 57571 & 40,04 & 46177 & 36,58 & 51874 & 38,42 \\
\hline ISS EPS & 26897 & 18,70 & 14748 & 11,68 & 20822,5 & 15,42 \\
\hline CAFESALUDD EPS & 16439 & 11,43 & 14793 & 11,72 & 15616 & 11,57 \\
\hline HIMMANA VIVR SA EPS & 21390 & 14,89 & 21683 & 17,18 & 21536,5 & 15,95 \\
\hline CAJANAL & 2875 & 2,00 & - & & 1437,5 & 100 \\
\hline Total & 143793 & 100 & 126244 & 100 & 135018,5 & 1,06 \\
\hline
\end{tabular}

Fuente: Ministerio de la Protección Social - listados de afiliados a EPS y ARS 2003 y 2004. Los porcentajes y promedios fueron adicionados por el Grupo ${ }^{\text {. }}$

$\mathrm{La}$ anterior tabla confirma que efectivamente SALUDCOOP, ISS y CAFESALUD, en su

\footnotetext{
${ }^{1}$ Además de los datos suministrados por el Ministerio de la Protección Social, incluimos la información de los inscritos en el Sistema de Selección de Beneficiarios (SISBEN) en Neiva, según cifras suministradas por la Secretaría Departamental de Salud, así: Afiliados 2003 (Niveles I, II y III): 138.614. Afiliados 2004 (Niveles I y II): 154.173. PROMEDIO 146.393,5
} 
orden, son las entidades con mayor número de afiliados en los periodos analizados, lo que conduce a determinar la proporción de tutelas vs. afiliados, a fin de establecer cuál es la EPS con mayor índice de tutelas en su contra. Esta proporción se realizó con la siguiente fórmula:

$t=\frac{\text { No. T K }}{\text { No. A }}$

Donde $t$ es la tasa numérica, $\mathrm{T}$ es el número de tutelas y A el número de afiliados, multiplicada por una constante $(\mathrm{K}=1000)$.

Tabla 1B. Tasa de incidencia de tutela por entidad según afiliados

\begin{tabular}{|l|r|}
\hline 1. CAJANAI & 63,3 \\
\hline 2.ISS BIP & 17 \\
\hline 3.SOISALUD E.PS. S.A. & 11,9 \\
\hline 4. EPS SALUDCOOP & 9 \\
\hline 5. CAIBSAIUD ARS + BPS & 6,4 \\
\hline
\end{tabular}

Como se evidencia en la anterior tabla, a pesar de que CAJANAL'no figuraba entre el grupo que más presenta afiliados y tutelas en su contra, una vez aplicada la fórmula para determinar la tasa, resulta que CAJANAL es la entidad en Neiva que más tutelas presenta en relación con su número de afiliados.

En efecto, ante la cantidad de afiliados que tiene esta entidad, el número de tutelas es el más alto pues por cada 1.000 afiliados 63,3 presentan acciones de tutela, mientras que en SALUDCOOP, que ocupó el primer lugar en las cifras objetivas (Tabla 1), 9 de cada 1.000 afiliados presentan esta acción. En el ISS, por su parte, por cada 1.000 afiliados, 17 formulan reclamaciones de tutela.

Similar distribución porcentual se encuentra al comparar el presente estudio con el análisis sobre el comportamiento de los recobros a nivel nacional ${ }^{2}$, como se ilustra a continuación:

\section{"Porcentaje de participación en el valor total recobrado por tutelas y valor Per Cápita por empresa aseguradora. Datos recobro 2002"}

\begin{tabular}{|c|c|c|}
\hline $\begin{array}{c}\text { EMPRESA ASEGURADORA } \\
\text { QUE RECOBRÓ } \\
\text { POR TUTELIAS }\end{array}$ & $\begin{array}{c}\text { \% DE PARTICIPACIÓN } \\
\text { DE CADA EMPRESA } \\
\text { EN EL } 2002\end{array}$ & $\begin{array}{c}\text { VALOR } \\
\text { PER CÁPTIA }\end{array}$ \\
\hline \begin{tabular}{|l|} 
SALUDCOOP \\
\end{tabular} & 20,77 & 4.638 \\
\hline SUSALUD & 17,50 & 11.761 \\
\hline COOMEVA & 8,54 & 3.158 \\
\hline SEGURO SOCIAL & 8,14 & 1.338 \\
\hline CATANAL & 7,80 & 9.916 \\
\hline CAFESALUD & 6,95 & 4.951 \\
\hline SÁNTTAS & 6,37 & 6.707 \\
\hline COLMÉDICA & 5,95 & 11.304 \\
\hline FAMISANAR & 3,93 & 3.153 \\
\hline CRUZ BLANCA & 2,84 & 2.972 \\
\hline COMPENSAR & 1,83 & 2.083 \\
\hline COMFENALCO & 1,79 & 2.517 \\
\hline SOS & 1,59 & 2.429 \\
\hline SALUDTOTAL & 1,49 & 1.091 \\
\hline SOLSALUD & 1,12 & 3.800 \\
\hline OTRAS ${ }^{*}$ & 0,96 & 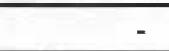 \\
\hline Total & 100,00 & \\
\hline
\end{tabular}

*Incluye Caprecom, Colseguros, Unimec, Humana, EPS Caldas, Emsanar, Salud Vida.

Comparando el anterior cuadro con las cifras de este estudio se observa que en lo regional, para los años 2003 a 2004, en lo que tiene que ver con la empresa SALUDCOOP, se repite la tendencia en cifras objetivas que se determinó para el año 2002 a nivel nacional, y en los lugares $4^{\circ}, 5^{\circ}$ y $6^{\circ}$. se ubicaron en su orden, el ISS, CAJANAL y CAFESALUD, de modo que la ubicación de estas entidades en la presente investigación es muy similar $\left(2^{\circ}\right.$., $3^{\circ}$. y $6^{\circ}$., respectivamente).

Este comportamiento se presenta igualmente en las fuentes del Ministerio de la Protección Social para el periodo 2001 - 2005, como se ilustra a continuación:

\footnotetext{
2 PINTO MASIS, Diana; CASTELLANOS, María Isabe. Caracterización de los recobros por tutelas y medicamentos no incluidos en los planes obligatorios de salud. En: Revista Gerencia y Políticas de Salud. Vol. 3, No. 7, Universidad Javeriana, Bogotá, 2004, p. 40.
} 
Causas, Manifestaciones y Efectos de la Vulneración del Derecho

“Recobro por Tutela y Comité Técnico Cientifico"

\begin{tabular}{|c|r|}
\hline Año & No. \\
\hline 2001 & 638 \\
\hline 2002 & 68.986 \\
\hline 2003 & 133.070 \\
\hline 2004 & 156.788 \\
\hline 2005 & 284.014 \\
\hline Total & 643.496 \\
\hline
\end{tabular}

Fuente: Ministerio de la Protección Social, abril de 2006.

Volviendo al análisis de la Tabla 1 se destaca la situación de la Secretaría Departamental de Salud. Esta dependencia registra el 7,3\% de rciones de tutela formuladas en su contra, lo que representa el $4^{\circ}$ puesto dentro de las más sccionadas; sin embargo, al determinarse la tasa de incidencia de tutela, resulta que por cada mil personas, menos de una $(0,7)$ formula esta acción judicial contra la Secretaría Departamental de Salud.

Esto se explica debido a la alta cantidad de personas inscritás en el SISBEN $(146.393,5)$, cifra superior en un $8,42 \%$ a la del régimen contributivo $(135.018,5)$.

Cabe anotar además, que los usuarios del régimen subsidiado valoran mejor los servicios del sistema de salud que los afiliados al régimen contributivo ${ }^{3}$.

Analizado el tema de los usuarios del régimen contributivo, a continuación se presentan los resultados de los demás usuarios.
Tabla 1C. Acciones ejercidas por usuarios vinculados o subsidiados:

\begin{tabular}{|l|c|c|}
\hline SECRESALUD DPTAL & 112 & 7,3 \\
\hline SOISALUD & 067 & 4,4 \\
\hline CAJA SALUD & 024 & 1,6 \\
\hline CAPRECOM & 017 & 1,1 \\
\hline COMFAMULIAR ARS & 014 & 1,0 \\
\hline SECRESALUD MPAL & 014 &, 9 \\
\hline ASMET SALUD & 011 &, 7 \\
\hline ECOOPSOS & .003 &, 2 \\
\hline Total & $\mathbf{2 6 2}$ & $\mathbf{1 7 , 2}$ \\
\hline
\end{tabular}

Para el análisis del régimen subsidiado o vinculado, se recuerda que su número de afiliados es mayor al del contributivo, y son los usuarios contributivos quienes más formulan acciones de tutela. Por ello, sólo el 17,14\% del total de acciones de amparo pertenece al régimen subsidiado o vinculado, a pesar de que esta población es la mayoritaria.

Se destaca el caso de SOLSALUD, que en esta investigación presenta el segundo lugar con mayor número de accionantes en su contra, situación que coincide con los resultados de la investigación nacional efectuada por la Defensoría del Pueblo al evaluar los servicios de las prestatarias ${ }^{4}$ para el año 2003, donde esta ARS se ubicó dentro de los puntajes más bajos en satisfacción del usuario.

Agotada la presentación del análisis sobre las prestatarias, corresponde ahora continuar con la caracterización sobre los accionantes o afectados.

\footnotetext{
${ }^{3}$ Defensoría del Pueblo, Evaluación de los servicios de salud que brindan las EPS 2005. Bogotá: Imprenta Nacional, 2005, pág. 111

${ }^{4}$ Tbíd. Pág. 114.
} 


\subsection{Características Relacionadas con el Afectado}

El impacto que ha representado la acción de tutela en el ciudadano, como institución eficaz para su acercamiento a la justicia, exige analizar cualitativamente los sujetos que acuden a ésta, y con ello establecer las causas y características de dichampoblación que, como se dijo ${ }^{5}$, es la mayoritaria en Colombia.

Para este fin, se presenta a continuación los resultados y la evaluación sobre el afectado según su género, edad, tipo de petición y representante judicial.

\subsection{El género}

Tabla 2. Afectado según genero

\begin{tabular}{|l|c|c|c|c|}
\hline Género & $\begin{array}{c}\text { Promedio } \\
\text { accionantes }\end{array}$ & $\%$ & $\begin{array}{c}\text { Población de Neiva } \\
\text { promedio }\end{array}$ & $\%$ \\
\hline Hombre & 379 & 49,7 & 179.076 & 47,5 \\
\hline Mujer & 384 & 50,3 & 197.648 & 52,5 \\
\hline Total & 763 & 100 & 376.724 & .100 \\
\hline
\end{tabular}

Teniendo en cuenta que la mayor cantidad de afectados que acudieron a la acción de tutela corresponde a las mujeres, se puede observar que existe un equilibrio con los afectados hombres, con una variación de apenas el $0,3 \%$. Hubiese sido recomendable comparar esta proporción con la distribución entre afiliados, pero no fue posible porque los datos suministrados por el Ministerio de la Protección Social no incluyen el criterio del género.
Si se analizan las cifras de género con el objeto de la petición, resulta que entre las tres situaciones de mayor reclamación entre los adultos $(68,1 \%)$, la distribución entre peticiones de hombres y de mujeres es la siguiente:

\section{Tabla 2A. Principales peticiones de adultos por género}

\begin{tabular}{|l|r|r|}
\hline \multicolumn{1}{|c|}{ Petición } & \% hombres & \% mujeres \\
\hline Medicamentos & 37,7 & 62,3 \\
\hline Tratami ento quirúrgico & 41 & 59 \\
\hline Tratamiento no quirúrgico & 50 & 50 \\
\hline
\end{tabular}

En cambio, entre la población de discapacitados, la solicitud de los hombres es mayor $(84,2 \%)$ que la de las mujeres $(15,8 \%)$, al igual que en la población de menores, neonatos yreciénnacidos, donde el $55,5 \%$ de las acciones corresponde a las de los hombres y el $44,4 \%$ es de las mujeres. Esta constante se mantiene incluso en el caso de la tercera edad, con una relación del $58,1 \%$ de peticiones para los hombres, y del $42 \%$ para las mujeres.

El comportamiento de la población por género en las peticiones minoritarias, que representan el $31,9 \%$ del total de reclamos, se muestra en la tabla No. 1 de los anexos.

\subsubsection{Afectado según edad}

Tabla 3 : Descripción del afectado por edad

\begin{tabular}{|l|c|c|c|c|}
\hline $\begin{array}{c}\text { Accionantes } \\
\text { Grupos etáreos }\end{array}$ & $\begin{array}{c}\text { Promedio } \\
\text { accionantes }\end{array}$ & $\begin{array}{c}\text { Acrionantes } \\
\%\end{array}$ & $\begin{array}{c}\text { Promedio } \\
\text { población Neiva } \\
2003-2004^{7}\end{array}$ & $\begin{array}{c}\text { Población } \\
\text { Neiva } \\
\%\end{array}$ \\
\hline $\begin{array}{l}\text { Entre } 18 \text { y } 60 \\
\text { años }\end{array}$ & 464,5 & 62 & 172.264 & 45,73 \\
\hline Entre 1 a 18 años & 175 & 22,9 & 174.487 & 46,32 \\
\hline Mayor a 60 años & 108,5 & 14,2 & 20.835 & 5,53 \\
\hline
\end{tabular}

\footnotetext{
${ }^{5}$ LOPEZ DAZA, German Alfonso. La Justicia Constitucional Colombiana ¿Un gobierno de los jueces? Neiva: Editorial Universidad Surcolombiana, 2005. p. 250

${ }_{7}^{6}$ DANE, 2006

7 FUENTE: Cálculos Departamento Administrativo de Planeación, POBLACION ESTIMADA URBANA Y RURAL DEL MUNICIPIO DE NEIVA PERIODO 2000 - 2005. Neiva, 2006
} 


\begin{tabular}{|l|c|c|c|c|}
\hline Entre 0 y 30 dias & 5 &, 7 & $0-1$ año: 9.105 & 2.41 \\
\cline { 1 - 3 } $\begin{array}{l}\text { Entre 1 y } 12 \\
\text { meses }\end{array}$ & 1,5 & 2 & & \\
\hline Total & & 100 & 376.691 & 100 \\
\hline
\end{tabular}

De los anteriores datos se concluyen las siguientes observaciones:

- Se conserva una proporción constante entre las edades de accionantes y de la población local pues los adultos, por ejemplo, son el grupo mayoritario en ambos casos.

- Sin embargo, los accionantes entre 1 y 18 años de edad representan un $22,9 \%$ del total de demandantes, mientras que su incidencia poblacional en Neiva es superior $(46,32 \%)$, lo que indica que la relación entre habitantes y accionantes de menor edad es inversamente proporcional. Se concluye que éste es el grupo que menos acude a la acción de tutela.

- En la misma tendencia el grupo de los niños entre un día de nacidos y los doce meses de edad correspónde a menos del $1 \%(0,9)$ de los accionantes, mientras que el mismo grupo equivale al $2,41 \%$ de la población de Neiva.

Para estas dos observaciones previas, desde el punto de vista normativo, se destaca que en el campo del menor, el artículo 44 de la Constitución Política consagra los derechos a la salud y a la seguridad social como derechos fundamentales de protección a la niñez, y por lo mismo, de protección directa e inmediata.

Los menores son las primeras personas que tenen protección particular por parte del Estado.

Ellos son sujetos especiales y diferentes a los -lultos, ya que son seres humanos en proceso de formación y en virtud de encontrarse en etapa de desarrollo, tienen unas condiciones de inferioridad frente a los demás.

La minoridad (consagrada con la categoría de "niño" en la Constitución Política en el artículo 44 y como "menor" en el artículo 68 inc. 4) se encuentra determinada por la edad fijada legalmente en los 18 años. La misma Constitución en el parágrafo del artículo 98 establece que la ciudadanía se adquiere con el cumplimiento de los dieciocho años de edad.

Así mismo, la Convención de los Derechos del Niño acogida como legislacjón interna mediante la Ley 12 de 1991, establece en su artículo $1^{\circ}$ que niño es todo ser humano menor de dieciocho años, salvo que, en virtud de la ley que le sea aplicable, haya alcanzado antes la mayoría de edad.

De otra parte, si se trata de encontrar el sustento normativo para establecer las diferentes categorías de menores de edad (neonato, recién nacido, infante, niño, adolescente, etc.) no existe norma legal o constitucional que lo establezca. En consecuencia, es necesario acudir a la ciencia para hacer claridad sobre el tema.

La enciclopedia médica $\operatorname{ESPASA}^{8}$ define al neonato como el ser humano recién nacido, al niño como aquel ser humano comprendido entre los dos y los doce años de edad, el adolescente como el ser humano cuyo periodo se encuentra comprendido entre el inicio de la pubertad y la terminación del desarrollo físico, y la pubertad como el período en el cual se van desarrollando los caracteres secundarios y se alcanza la capacidad genésica.

Ya en el campo legislativo y jurisprudencial, el menor hoy día goza de una amplia protección,

Fociclopedia médica ESPASA ESCALPE, redacción y realización electrónica: Planeta Actimedia, S.A. Instituto - aífico y Tecnológico de la Universidad de Navarra, 2000 
tanto por la normatividad, como por la jurisprudencia. En la actualidad el menor tiene una protección integral externa (ultrafamiliar) para el desarrollo o su protección misma' ${ }^{9}$.

La normatividad colombiana que protege al menor se encuentra principalmente en el Código del Menor (Decreto 2737 de 1989), reglamentación expedida con anterioridad a la Carta Pôlítica de 1991 y a la Convención de los Derechos del Niño del 20 de noviembre de 1989, acogida como normatividad interna mediante Ley 12 de 1991.

Este Código del Menor adoptó una política de protección integral de los menores, consagrando y garantizando sus derechos individualmente considerados tanto en situaciones de normalidad, como en situaciones irregulares para prevenir y corregir. Además, el artículo 238 de la Ley 50 de 1990 consagra el descanso remunerado de la madre durante la lactancia.

La Carta de 1991 establece algunas protecciones especiales que se le deben al menor, tales como las consagradas en el artículo 50 que prevé la protección o seguridad social del menor de un año cuanto no esté cubierto, o-el derecho de los niños de 5 años a cursar la etapa preescolar (artículo 67 inc. $3^{\circ}$ ); o la del niño entre los 5 y 15 años a cursar los nueve niveles escolares básicos; la del adolescente que requiere protección integral (artículo 45 inc. $1^{\circ}$ ) y la de los jóvenes que necesitan de protección, educación y progreso (artículo 45 inc.2).

Por lo tanto, el derecho colombiano reconoce que los menores de edad merecen una especial protección, debido a que corresponden a uno de los grupos más vulnerables, y por sus condiciones de subordinación e indefensión. Estos dos criterios se han definido de la siguiente forma: ${ }^{10}$
Subordinación: Condición de una persona sujeta a otra o dependiente de ella.

Indefensión: Se produce cuando una persona sin culpa de su parte, no ha podido defenderse o defender sus derechos conforme a las leyes que reglamentan su ejercicio.

Existiendo las protecciones ya mencionadas, para el presente estudio se critica que sea la población de los menores la segunda mayor afectada. Este grupo comprende a las personas entre 1 y 18 años de edad, y en estos resultados incluso están por encima de las personas discapacitadas y de la tercera edad.

Sin embargo, dentro del grupo de menores de edad del análisis llama la atención el bajo índice de afectados entre 0 y 12 meses de nacidos, que equivale a menos del $1 \%(0,9)$ lo que resulta paradójico cuando ellos pertenecen al grupo de los menores de edad, que tiene las protecciones jurídicas específicas ya referidas. Las prestatarias entonces, tienen una fuente clara y directa para asumir su función, de modo que es cuestionable que la acción de tutela se haya convertido en un mecanismo o trámite administrativo.

\subsection{Solicitudes Presentadas en las acciones de Tutela}

Las solicitudes son entendidas en este estudio como el objeto de la petición del accionante en la tutela, las cuales para esta investigación tienen el siguiente contenido ${ }^{11}$ :

- Medicamento: Producto que administrado en el organismo coopera con éste en la recuperación de la Salud.

- Tratamiento no quirúrgico: Intervenciones en las que no se utilizan métodos quirúrgicos o invasivos.

\footnotetext{
${ }^{9}$ LAFONT PIANETTA, Pedro. Derecho de Familia. Bogotá: Ediciones Librería del Profesional, 1997, p.447

${ }^{10}$ Corte Constitucional, Sentencia T-197/93, M.P. Alejandro Martínez Caballero

${ }^{11}$ Diccionario ESPASA de Medicina, Op. Ct.
} 
- Cirugía: La que se caracteriza por el empleo principal de métodos quirúrgicos.

- Alto costo: Conforme al artículo 17 de la Resolución 5261 de 1994 del Ministerio de Salud, y en concordancia con el Decreto 806 de 1997, se consideran como alto costo o enfermedades ruinosas o catastróficas, "aquellos tratamientos utilizados en el manejo de enfermedades ruinosas o catastróficas que se caracterizan por un bajo costo efectividad en la modificación del pronóstico y representan un alto costo".

Se incluyen los siguientes:

a. Tratamiento con radioterapia y quimioterapia para el cáncer.

b. Diálisis para insuficiencia renal crónica, transplante renal, de corazón, de medula ósea y de cornea.

c. Tratamiento para el SIDA y sus complicaciones.

d. Tratamiento quirúrgico para enfermedades del corazón y del sistema nervioso central.

e. Tratamiento quirúrgico para enfermedades de origen genético o congénitas.

f. Tratamiento medico quirúrgico para el trauma mayor.

g. Terapia en unidad de cuidados intensivos.

h. Reemplazos articulares.

Sin embargo, para efectos de este análisis, el tratamiento para el SIDA se dejó como un ítem independiente, debido a su alta incidencia social y al especial tratamiento jurisprudencial que ha tenido este tema.

- Prótesis: Sustitución de una parte del esqueleto o de un órgano por una pieza o implante especial que reproduce más o menos lo que ha de sustituir.

- Ortesis: Férulas, herrajes y soportes. Dispositivo utilizado para proteger, soportar o mejorar la función de segmentos del cuerpo que se mueven.

- Exámenes paraclínicos: Métodos de investigación por medios físicos o sicológicos para llegar al diagnóstico de un paciente.

- SIDA: Síndrome de Inmunodeficiencia Adquirida.

En la siguiente tabla se presentan otro tipo de solicitudes no definidas aquí pues son conceptos de común uso, y además tienen bợja incidencia en los resultados.

Tabla 4 Solicitud

\begin{tabular}{|l|c|c|c|}
\hline \multicolumn{1}{|c|}{ Concepto } & Frecuencia & $\%$ & $\begin{array}{c}\text { Resultados a nivel } \\
\text { nacional año } \\
2003 \%^{12}\end{array}$ \\
\hline Medicamento & 488 & 32 & 17,9 \\
\hline Tratamiento no quirúrgico & 321 & 21 & $8,9^{13}$ \\
\hline Cirugía & 219 & 14,3 & 16,8 \\
\hline Alto costo (no incluye SIDA) & 201 & 13,1 & ND $^{14}$ \\
\hline Prótesis y ortesis & 111 & 7,3 & 5,9 \\
\hline Exámenes paraclínicos & 89 & 5,8 & 23,6 \\
\hline Sida & 34 & 2,2 & 11,7 \\
\hline Otros & 67 & 4,3 & \\
\hline Total & 1530 & 100,0 & \\
\hline
\end{tabular}

Otros: Afiliación (1,4\%), pago de incapacidad $(1,4 \%)$, reembolso $(0,8 \%)$, gastos de desplazamiento $(0,2 \%)$, licencia de maternidad $(0,1 \%)$, pensión de invalidez $(0,1 \%)$, indemnización $(0,1 \%)$, lentes $(0,1 \%), \quad y$ multiafiliación $(0,1 \%)$.

Fuente: Defensoría del Pueblo, Causas de la tutela en salud Bogotá: 2004, página 19.

Para obtener este porcentaje se excluyeron los datos de alto costo y sida de la fuente originaria (págs. 19 y 26 de la fuente citada).

${ }^{14}$ En la fuente originaria hubo una metodología y variables diferentes que implicó que los tratamientos de alto costo se analizaran en múltiples tablas cuyos parámetros en este aspecto no se pueden equiparar a nuestra metodología. 
En el nivel naciona ${ }^{15}$ las solicitudes con mayor porcentaje son los exámenes paraclínicos $(23 \%)$, seguidos de las cirugías (16,8\% para 2003), y las reclamaciones de medicamentos ocupan el cuarto lugar con $17,9 \%$. En el nivel local se observa, que la tendencia para los años 2003 a 2004 es inversa, ya que la mayor reclamación se generó por concepto de medicamentos, con un $32 \%$.

Esta cifra de los hallazgos en Neiva coincide con la problemática nacional actual, ya que a raíz de las discusiones que se llevaron a cabo en la pasada legislatura con motivo de la fallida reforma a la Ley 100 de 1993, el Gobierno se mostró preocupado por el alto número de tutelas en materia de medicamentos, específicamente sobre los que no están contemplados en el $\mathrm{POS}^{16}$, por lo que el Viceministro Técnico del Ministerio de la Protección Social refirió que muchos de los medicamentos son fabricados por monopolios, y que en muchos casos no es necesario recurrir a los productos de marca cuando se pueden utilizar los que sí están incluidos en el POS,

Indicó el Viceministro que "entree el 2001 y el 2004 se registró un incremento del $350 \%$ en el número de tutelas para exigir medicamentos de alto costo, a pesar de que existen alternativas cubiertas por el plan, que sirven para tratar la enfermedad".

Si bien en la presente investigación se confirma este primer lugar local para los medicamentos, no es posible determinar que ellas correspondan al alto costo, pues en la muestra no se discriminó este indicador.
Sin embargo, en materia de medicamentos se observa que las reclamaciones en Neiva versan principalmente sobre los siguientes: algunos antiretrovirales, ciclos de tratamientos para quimioterapia y para esclerosis múltiple. Como novedad, y siguiendo la tendencia nacional ante la jurisprudencia de la Corte Constitucional ${ }^{17}$, se vienen acrecentando las reclamaciones del medicamento Sildenafic (Viagra) por disfunciones sexuales, principalmente reclamadas por los afiliados a CAJANAL.

De otra parte, en esta investigación se determinó que el $65,3 \%$ de las solicitudes se presentaron sobre reclamaciones distintas a los tratamientos quirúrgicos, a las enfermedades de alto costo y al suministro de prótesis u órtesis, es decir que el porcentaje mayoritario de reclamos versa sobre actividades no quirúrgicas o de alta complejidad.

\section{CONCLUSIONES}

- Resulta contradictorio que sean las EPS las que presentan los mayores índices de tutelas en su contra, siendo que ellas reciben mayores recursos para la atención a sus usuarios, los cuales además, son menos que los que pertenecen al régimen subsidiado. Lo esperado sería que quienes más tutelan fueran los pobres, por no estar afiliados al régimen contributivo y ser la mayoría de usuarios del sistema. Esta contradicción es reprochable y lleva a que se cuestione el ánimo de lucro no social con que se administran estas EPS, ante su naturaleza privada.

\footnotetext{
${ }^{15}$ Tbíd.

${ }^{16}$ CAMARGO MANTIVA, Adriana. Denuncian negocio con tutelas de salud. En: El Tiempo, Bogotá: 18 de octubre de 2005; p. 2 A.

${ }^{17}$ La Sentencia fundadora que incluyó por primera vez la tutela de los derechos a la dignidad y la salud por la negativa al suministro de este medicamento es la T-926 de 1999, M.P. Carlos Gaviria Díaz, donde se resolvió el caso de un paciente de una EPS de Neiva por una disfunción sexual originada en su diabetes. Luego se profirió la sentencia T-465 de 2002, M.P. Marco Gerardo Monroy Cabra.
} 
- CAJANAL y el ISS son entidades de naturaleza pública y ellas agrupan la mayor cantidad de accionantes por afiliado $(80,70 \%)$, y por ello se cuestiona la intervención del Estado en las áreas de salud como administradores y prestatarios, ya que no se evidencia una correcta gestión de los recursos que reciben para la prestación del servicio.

- Siendo SALUDCOOP una empresa del sector solidario, regulada como tal con unos principios que vinculan activamente la ausencia de ánimo de lucro, al evaluar su comportamiento en la reclamación de tutela de sus usuarios es contradictorio que ocupe el segundo lugar entre las empresas de naturaleza privada contra las cuales se formulan estas acciones.

- Los bajos índices de acciones de tutela formuladas por usuarios del régimen subsidiado y vinculado no se justifican en el desconocimiento de estos afectados sobre la institución de la tutela, pues ya se había establecido en investigación nacional previa $^{18}$, qưe su nivel de satisfacción sobre la calidad del sistema de salud es muy superior al de los afiliados al régimen contributivo.

- La mayoría de las prestatarias del servicio de salud no aplican en debida forma los imperativos constitucionales que ordenan la protección directa, inmediata y privilegiada al grupo de los menores de edad, porque esta población es la segunda más afectada.

- La Personería Municipal y la Defensoría del Pueblo ejercen un papel activo en su contacto y su asesoría al usuario, situación que no se presenta con la Procuraduría General de la Nación que interviene más en la sugerencia de políticas legislativas.

- La Superintendencia Nacional de Salud por su parte, si bien ejerce las funciones constitucionales y legales de vigilar y controlar las actuaciones de lasprestadoras del servicio, no ha llegado a convertirse en punto de referencia del ciudadano común que ve vulnerado su derecho a la salud, y a ello puede contribuir la excesiva centralización de su sede, puesto que en la ciudad de Neiva no existe oficina regional para este propósito.

- El alto porcentaje de decisiones de los jueces de Neiva declarando la conexidad del derecho a la salud con el derecho a la vida implica que el juez interviene en políticas públicas para la satisfacción del derecho fundamental en cuanto a la consecución de gastos, cuando ordena a la administración tal gestión.

${ }^{18}$ Defensoría del Pueblo, 2005, Op. Cit., págs. 90 a 108 